\title{
Retrospective-Cost Adaptive Control of Uncertain Hammerstein Systems Using a NARMAX Controller Structure
}

\author{
Jin Yan * Anthony M. D’Amatoł and Dennis S. Bernstein ${ }^{\ddagger}$ \\ University of Michigan, 1320 Beal Ave., Ann Arbor, MI 48109
}

\begin{abstract}
We apply an extension of retrospective cost adaptive control (RCAC) to a commandfollowing problem for uncertain Hammerstein systems. In particular, RCAC with a NARMAX controller strucuture is applied to linear systems cascaded with input nonlinearities. We assume that one Markov parameter of the linear plant is known. RCAC also uses knowledge of the monotonicity properties of the input nonlinearity. The goal is to determine whether RCAC with a NARMAX controller structure can improve the command-following performance compared to the linear RCAC controller.
\end{abstract}

\section{Introduction}

Many practical systems can be modeled as linear systems cascaded with input and output nonlinearities. Systems with input nonlinearities are called Hammerstein systems. Examples of memoryless nonlinearities include saturation and deadzone, while nonlinearities with memory include hysteretic actuators and sensors. Identification and control techniques have been extensively developed for these systems. ${ }^{1-3,5}$

In practice, however, the linear component of the system as well as the nonlinearities may be uncertain. In this case, robust control techniques can be used. ${ }^{4}$ However, adaptive control methods may be desirable to allow the controller to tune itself to the actual plant characteristics, especially when unexpected changes can occur during plant operation.

In recent research ${ }^{6-8}$ we demonstrated the ability of retrospective cost adaptive control (RCAC $)^{9-14}$ to control systems involving linear dynamics with input and output nonlinearities. In all of these papers the goal is to adapt an instantaneously linear controller for a nonlinear plant. Although RCAC is able to tune the linear controller to the command signal and nonlinear characteristics of the plant, the ability of the linear controller to produce accurate command following is limited by the distortion introduced by the nonlinearities. The objective of the present paper is to develop a technique for reducing this distortion.

The approach that we take in the present paper is to replace the linear controller structure of RCAC by a nonlinear controller structure. A simple and effective way to do this is to use a NARMAX (nonlinear ARMAX) controller structure that is linear in parameters. NARMAX models have been used extensively for system identification ${ }^{15-17}$ and as a plant model for adaptive control. ${ }^{18}$ The approach of the present paper differs from prior work by using a NARMAX model structure for the adaptive controller itself, where the nonlinearities are chosen prior to controller implementation and the controller coefficients are updated online by RCAC.

${ }^{*}$ Graduate Student, Aerospace Engineering Department

${ }^{\dagger}$ NASA GSRP Fellow, Aerospace Engineering Department

${ }_{\ddagger}^{\ddagger}$ Professor, Aerospace Engineering Department 
The controller nonlinearities can be applied to either the input to the controller (NARMAX/I), the output of the controller (NARMAX/O), or both (NARMAX/IO). The choice of NARMAX controller structure is chosen to reflect the presence of the unknown input or output nonlinearities in the plant with the goal of at least partially inverting these nonlinearities to reduce the distortion that degrades the commandfollowing accuracy. In the present paper we investigate various choices of the controller nonlinearities in order to determine their effectiveness in improving the closed-loop performance. The goal is to determine controller nonlinearities that are effective for large classes of uncertain input nonlinearities.

The contents of the paper are as follows. In Section II, we describe the Hammerstein commandfollowing problem. In Section III, we apply the NARMAX controller structure to Hammerstein systems. In particular, we apply an extension of RCAC using auxiliary nonlinearities, and employ a nonlinear controller structure to reduce the command-following distortion introduced by the input nonlinearities. Numerical results are also presented in Section IV, and conclusions are given in Section V.

\section{Hammerstein Command-following Problem}

Consider the SISO discrete-time Hammerstein system

$$
\begin{aligned}
x(k+1) & =A x(k)+B \mathcal{N}(u(k)), \\
y(k) & =E_{1} x(k),
\end{aligned}
$$

where $x(k) \in \mathbb{R}^{n}, u(k), y(k) \in \mathbb{R}, w(k) \in \mathbb{R}^{d}, \mathcal{N}: \mathbb{R} \rightarrow \mathbb{R}$, and $k \geq 0$. We consider the Hammerstein command-following problem with performance variable

$$
z(k)=y(k)-r(k)
$$

where $z(k), r(k) \in \mathbb{R}$. The goal is to develop an adaptive output feedback controller that minimizes the command-following error $z$ with minimal modeling information about the dynamics, and input nonlinearity $\mathcal{N}$. We assume that measurements of $z(k)$ are available for feedback; however, measurements of $v(k)=$ $\mathcal{N}(u(k))$ are not available. A block diagram for (1)-(3) is shown in Figure 1.

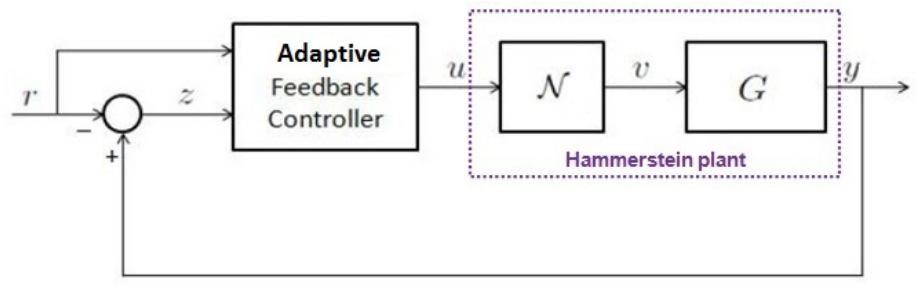

Figure 1. Adaptive command-following problem for a Hammerstein plant. We assume that measurements of $z(k)$ are available for feedback; however, measurements of $v(k)=\mathcal{N}(u(k))$ is not available. The feedforward path is optional.

\section{Retrospective-Cost Adaptive Control}

\section{III.A. Retrospective Cost with Adaptive Regularization}

For $i \geq 1$, define the Markov parameter

$$
H_{i} \triangleq E_{1} A^{i-1} B
$$

For example, $H_{1}=E_{1} B$ and $H_{2}=E_{1} A B$. Let $\ell$ be a positive integer. Then, for all $k \geq \ell$,

$$
x(k)=A^{\ell} x(k-\ell)+\sum_{i=1}^{\ell} A^{i-1} B \mathcal{N}((u(k-i)),
$$


and thus

$$
z(k)=E_{1} A^{\ell} x(k-\ell)+\bar{H} \bar{V}(k-1)-r(k),
$$

where

$$
\bar{H} \triangleq\left[\begin{array}{ccc}
H_{1} & \cdots & H_{\ell}
\end{array}\right] \in \mathbb{R}^{1 \times \ell}
$$

and

$$
\bar{V}(k-1) \triangleq\left[\begin{array}{c}
\mathcal{N}(u(k-1)) \\
\vdots \\
\mathcal{N}(u(k-\ell))
\end{array}\right] .
$$

Next, we rearrange the columns of $\bar{H}$ and the components of $\bar{V}(k-1)$ and partition the resulting matrix and vector so that

$$
\bar{H} \bar{V}(k-1)=\mathcal{H}^{\prime} V^{\prime}(k-1)+\mathcal{H} V(k-1),
$$

where $\mathcal{H}^{\prime} \in \mathbb{R}^{1 \times\left(\ell-l_{V}\right)}, \mathcal{H} \in \mathbb{R}^{1 \times l_{V}}, V^{\prime}(k-1) \in \mathbb{R}^{\ell-l_{V}}$, and $V(k-1) \in \mathbb{R}^{l_{V}}$. Then, we can rewrite (5) as

$$
\begin{gathered}
z(k)=\mathcal{S}(k)+\mathcal{H} V(k-1), \\
\mathcal{S}(k) \triangleq E_{1} A^{\ell} x(k-\ell)-r(k)+\mathcal{H}^{\prime} V^{\prime}(k-1) .
\end{gathered}
$$

Next, for $j=1, \ldots, s$, we rewrite (7) with a delay of $k_{j}$ time steps, where $0 \leq k_{1} \leq k_{2} \leq \cdots \leq k_{s}$, in the form

$$
z\left(k-k_{j}\right)=\mathcal{S}_{j}\left(k-k_{j}\right)+\mathcal{H}_{j} V_{j}\left(k-k_{j}-1\right),
$$

where (8) becomes

$$
\mathcal{S}_{j}\left(k-k_{j}\right) \triangleq E_{1} A^{\ell} x\left(k-k_{j}-\ell\right)+\mathcal{H}_{j}^{\prime} V_{j}^{\prime}\left(k-k_{j}-1\right)-r\left(k-k_{j}\right)
$$

and (6) becomes

$$
\bar{H} \bar{U}\left(k-k_{j}-1\right)=\mathcal{H}_{j}^{\prime} V_{j}^{\prime}\left(k-k_{j}-1\right)+\mathcal{H}_{j} V_{j}\left(k-k_{j}-1\right),
$$

where $\mathcal{H}_{j}^{\prime} \in \mathbb{R}^{1 \times\left(\ell-l_{V_{j}}\right)}, \mathcal{H}_{j} \in \mathbb{R}^{1 \times l_{V_{j}}}, U_{j}^{\prime}\left(k-k_{j}-1\right) \in \mathbb{R}^{\ell-l_{V_{j}}}$, and $V_{j}\left(k-k_{j}-1\right) \in \mathbb{R}^{l_{V_{j}}}$. Now, by stacking $z\left(k-k_{1}\right), \ldots, z\left(k-k_{s}\right)$, we define the extended performance

$$
Z(k) \triangleq\left[\begin{array}{c}
z\left(k-k_{1}\right) \\
\vdots \\
z\left(k-k_{s}\right)
\end{array}\right] \in \mathbb{R}^{s} .
$$

Therefore,

$$
Z(k) \triangleq \tilde{\mathcal{S}}(k)+\tilde{\mathcal{H}} \tilde{V}(k-1)
$$

where

$$
\tilde{\mathcal{S}}(k) \triangleq\left[\begin{array}{c}
\mathcal{S}_{1}\left(k-k_{1}\right) \\
\vdots \\
\mathcal{S}_{s}\left(k-k_{s}\right)
\end{array}\right] \in \mathbb{R}^{s},
$$


$\tilde{V}(k-1)$ has the form

$$
\tilde{V}(k-1) \triangleq\left[\begin{array}{c}
\mathcal{N}\left(\left(u\left(k-q_{1}\right)\right)\right. \\
\vdots \\
\mathcal{N}\left(u\left(k-q_{l_{\tilde{V}}}\right)\right)
\end{array}\right] \in \mathbb{R}^{l_{\tilde{V}}}
$$

where, for $i=1, \ldots, l_{\tilde{V}}, k_{1} \leq q_{i} \leq k_{s}+\ell$, and $\tilde{\mathcal{H}} \in \mathbb{R}^{s \times l_{\tilde{V}}}$ is constructed according to the structure of $\tilde{V}(k-1)$. The vector $\tilde{V}(k-1)$ is formed by stacking $V_{1}\left(k-k_{1}-1\right), \ldots, V_{s}\left(k-k_{s}-1\right)$ and removing copies of repeated components.

Next, for $j=1, \ldots, s$, we define the retrospective performance

$$
\hat{z}_{j}\left(k-k_{j}\right) \triangleq \mathcal{S}_{j}\left(k-k_{j}\right)+\mathcal{H}_{j} \hat{V}_{j}\left(k-k_{j}-1\right),
$$

where the past controls $V_{j}\left(k-k_{j}-1\right)$ in (9) are replaced by the retrospective controls $\hat{V}_{j}\left(k-k_{j}-1\right)$. In analogy with (10), the extended retrospective performance for (12) is defined as

$$
\hat{Z}(k) \triangleq\left[\begin{array}{c}
\hat{z}_{1}\left(k-k_{1}\right) \\
\vdots \\
\hat{z}_{s}\left(k-k_{s}\right)
\end{array}\right] \in \mathbb{R}^{s}
$$

and thus is given by

$$
\hat{Z}(k)=\tilde{\mathcal{S}}(k)+\tilde{\mathcal{H}} \hat{\tilde{V}}(k-1),
$$

where the components of $\hat{\tilde{V}}(k-1) \in \mathbb{R}^{l} \tilde{V}$ are the components of $\hat{V}_{1}\left(k-k_{1}-1\right), \ldots, \hat{V}_{s}\left(k-k_{s}-1\right)$ ordered in the same way as the components of $\tilde{V}(k-1)$. Subtracting (11) from (13) yields

$$
\hat{Z}(k)=Z(k)-\tilde{\mathcal{H}} \tilde{V}(k-1)+\tilde{\mathcal{H}} \hat{\tilde{V}}(k-1) .
$$

Finally, we define the retrospective cost function

$$
J(\hat{\tilde{V}}(k-1), k) \triangleq \hat{Z}^{\mathrm{T}}(k) R(k) \hat{Z}(k),
$$

where $R(k) \in \mathbb{R}^{s \times s}$ is a positive-definite performance weighting. The goal is to determine refined controls $\hat{\tilde{V}}(k-1)$ that would have provided better performance than the controls $U(k)$ that were applied to the system. The refined control values $\hat{\tilde{V}}(k-1)$ are subsequently used to update the controller.

Next, to ensure that (15) has a global minimizer, we consider the regularized cost

$$
\begin{aligned}
\bar{J}(\hat{\tilde{V}}(k-1), k) \triangleq & \hat{Z}^{\mathrm{T}}(k) R(k) \hat{Z}(k) \\
& +\eta(k) \hat{\tilde{V}}^{\mathrm{T}}(k-1) \hat{\tilde{V}}(k-1),
\end{aligned}
$$

where $\eta(k) \geq 0$. Substituting (14) into (16) yields

$$
\begin{aligned}
\bar{J}(\hat{\tilde{V}}(k-1), k)=\hat{\tilde{V}} & (k-1)^{\mathrm{T}} \mathcal{A}(k) \hat{\tilde{V}}(k-1) \\
& +\mathcal{B}(k) \hat{\tilde{V}}(k-1)+\mathcal{C}(k),
\end{aligned}
$$

where

$$
\begin{aligned}
& \mathcal{A}(k) \triangleq \tilde{\mathcal{H}}^{\mathrm{T}} R(k) \tilde{\mathcal{H}}+\eta(k) I_{l_{\tilde{V}}}, \\
& \mathcal{B}(k) \triangleq 2 \tilde{\mathcal{H}}^{\mathrm{T}} R(k)[Z(k)-\tilde{\mathcal{H}} \tilde{V}(k-1)], \\
& \mathcal{C}(k) \triangleq Z^{\mathrm{T}}(k) R(k) Z(k)-2 Z^{\mathrm{T}}(k) R(k) \tilde{\mathcal{H}} \tilde{V}(k-1) \\
& \quad+\tilde{V}^{\mathrm{T}}(k-1) \tilde{\mathcal{H}}^{\mathrm{T}} R(k) \tilde{\mathcal{H}} \tilde{V}(k-1) .
\end{aligned}
$$


If either $\tilde{\mathcal{H}}$ has full column rank or $\eta(k)>0$, then $\mathcal{A}(k)$ is positive definite. In this case, $\bar{J}(\hat{\tilde{V}}(k-1), k)$ has the unique global minimizer

$$
\hat{\tilde{V}}(k-1)=-\frac{1}{2} \mathcal{A}^{-1}(k) \mathcal{B}(k) .
$$

\section{III.B. NARMAX Controller Construction}

In this section, we assume a NARMAX structure for the adaptive controller, which uses a nonlinear difference equation to model the relation between the input $z$ and output $u$ of the controller. The nonlinear controller may include nonlinearities on the input to the controller (NARMAX/I), the output of the controller (NARMAX/O), or both (NARMAX/IO). The NARMAX controller structure is linear in the controller parameters, and linear regression is used to update the controller coefficients.

The control $u(k)$ is given by the strictly proper time-series controller of order $n_{\mathrm{c}}$ written as

$$
u(k)=\sum_{j=1}^{p} \sum_{i=1}^{n_{\mathrm{c}}} M_{j i}(k) f_{j}(u(k-i))+\sum_{j=1}^{q} \sum_{i=1}^{n_{\mathrm{c}}} N_{j i}(k) g_{j}(z(k-i)),
$$

where, for all $j=1, \ldots, p, i=1, \ldots, n_{\mathrm{c}}, M_{j i}(k) \in \mathbb{R}$, and $N_{j i}(k) \in \mathbb{R}$. The control (18) can be expressed as

$$
u(k)=\theta(k) \phi(k-1),
$$

where

$$
\begin{aligned}
& \theta(k) \triangleq\left[M_{11}(k) \cdots M_{1 n_{\mathrm{C}}}(k) M_{21}(k) \cdots M_{2 n_{\mathrm{C}}}(k) \cdots M_{p 1}(k) \cdots M_{p n_{\mathrm{C}}}(k)\right. \\
& \left.N_{11}(k) \cdots N_{1 n_{\mathrm{c}}}(k) N_{21}(k) \cdots N_{2 n_{\mathrm{c}}}(k) \cdots N_{q 1}(k) \cdots N_{q n_{\mathrm{c}}}(k)\right] \in \mathbb{R}^{l_{u} \times(p+q) n_{\mathrm{c}}}
\end{aligned}
$$

and

$$
\begin{aligned}
& \phi(k-1) \triangleq\left[f_{1}(u(k-1)) \cdots f_{1}\left(u\left(k-n_{c}\right)\right) \cdots \quad f_{p}(u(k-1)) \cdots f_{p}\left(u\left(k-n_{c}\right)\right)\right. \\
& \left.g_{1}(z(k-1)) \cdots g_{1}\left(z\left(k-n_{\mathrm{c}}\right)\right) \cdots g_{q}(z(k-1)) \cdots g_{q}\left(z\left(k-n_{\mathrm{c}}\right)\right)\right]^{\mathrm{T}} \in \mathbb{R}^{(p+q) n_{\mathrm{c}}} .
\end{aligned}
$$

To illustrate the NARMAX/I controller structure, let $f_{1}(u)=u, f_{2}(u)=u^{2}$, and $f_{3}(u)=u^{3}$. Then $\theta(k)$ and $\phi(k-1)$ can be expressed as

$$
\theta(k) \triangleq\left[M_{1}(k) \cdots M_{n_{\mathrm{c}}}(k) M_{n_{\mathrm{c}}+1}(k) \cdots M_{2 n_{\mathrm{c}}}(k) M_{2 n_{\mathrm{c}}+1}(k) \cdots M_{3 n_{\mathrm{c}}}(k) N_{1}(k) \cdots N_{n_{\mathrm{c}}}(k)\right] \in \mathbb{R}^{l_{u} \times n_{\mathrm{c}}\left(3 l_{u}+l_{z}\right)}
$$

and

$$
\phi(k-1) \triangleq\left[u(k-1) \cdots u\left(k-n_{c}\right) u^{2}(k-1) \cdots u^{2}\left(k-n_{c}\right) u^{3}(k-1) \cdots u^{3}\left(k-n_{c}\right) z(k-1) \cdots z\left(k-n_{c}\right)\right]^{\mathrm{T}} \in \mathbb{R}^{n_{c}\left(3 l_{u}+l_{y}\right)} .
$$

To illustrate the NARMAX/O controller structure, let $g_{1}(z)=z$ and $g_{2}(z)=z^{2}$. Then $\theta(k)$ and $g(\phi(k-1))$ can be expressed as

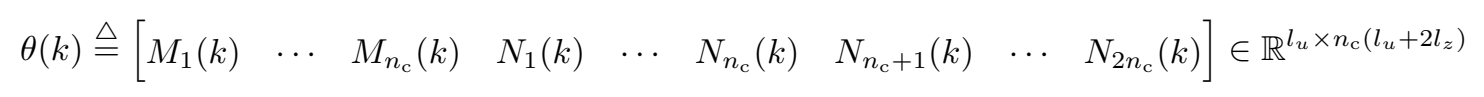

and

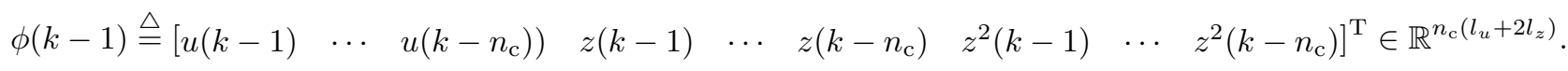
function

Next, let $d$ be a positive integer such that $\tilde{V}(k-1)$ contains $v(k-d)$ and define the cumulative cost

$$
J_{\mathrm{R}}(\theta, k) \triangleq \sum_{i=d+1}^{k} \lambda^{k-i}\left\|\phi^{\mathrm{T}}(i-d-1) \theta^{\mathrm{T}}(k)-\hat{v}^{\mathrm{T}}(i-d)\right\|^{2}+\lambda^{k}\left(\theta(k)-\theta_{0}\right) P_{0}^{-1}\left(\theta(k)-\theta_{0}\right)^{\mathrm{T}},
$$


where $\|\cdot\|$ is the Euclidean norm, and $\lambda \in(0,1]$ is the forgetting factor. Minimizing (19) yields

$$
\begin{aligned}
\theta^{\mathrm{T}}(k)= & \theta^{\mathrm{T}}(k-1)+\beta(k) P(k-1) \phi(k-d-1) \cdot\left[\phi^{\mathrm{T}}(k-d) P(k-1) \phi(k-d-1)+\lambda(k)\right]^{-1} \\
& \cdot\left[\phi^{\mathrm{T}}(k-d-1) \theta^{\mathrm{T}}(k-1)-\hat{v}^{\mathrm{T}}(k-d)\right],
\end{aligned}
$$

where $\beta(k)$ is either zero or one. The error covariance is updated by

$$
\begin{aligned}
P(k)= & \beta(k) \lambda^{-1} P(k-1)+[1-\beta(k)] P(k-1)-\beta(k) \lambda^{-1} P(k-1) \phi(k-d-1) \\
& \cdot\left[\phi^{\mathrm{T}}(k-d-1) P(k-1) \phi(k-d)+\lambda\right]^{-1} \cdot \phi^{\mathrm{T}}(k-d-1) P(k-1) .
\end{aligned}
$$

We initialize the error covariance matrix as $P(0)=\alpha I_{3 n_{c}}$, where $\alpha>0$. Note that when $\beta(k)=0, \theta(k)=$ $\theta(k-1)$ and $P(k)=P(k-1)$. Therefore, setting $\beta(k)=0$ switches off the controller adaptation, and thus freezes the control gains. When $\beta(k)=1$, the controller is allowed to adapt.

\section{III.C. Auxiliary Nonlinearities and the Adaptive NARMAX Controller}

To account for the presence of the input nonlinearity $\mathcal{N}$, the RCAC controller in Figure 2 uses two auxiliary nonlinearities. ${ }^{19}$ The auxiliary nonlinearity $\mathcal{N}_{1}$ modifies $u_{\mathrm{c}}$ to obtain the regressor input $u_{\mathrm{r}}$, while the auxiliary nonlinearity $\mathcal{N}_{2}$ modifies $u_{\mathrm{r}}$ to produce the Hammerstein plant input $u$. The auxiliary nonlinearities $\mathcal{N}_{1}$ and $\mathcal{N}_{2}$ are chosen based on limited knowledge of the input nonlinearity $\mathcal{N}$, as described below.

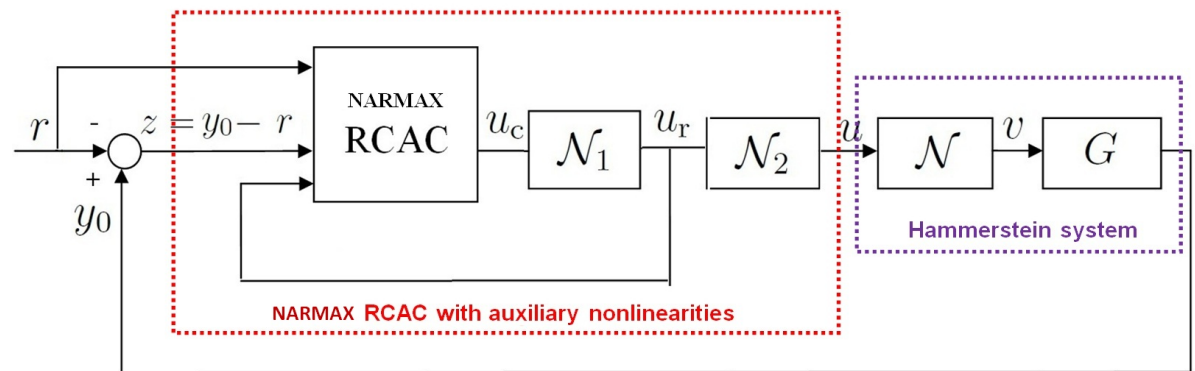

Figure 2. Hammerstein command-following problem with the NARMAX RCAC adaptive controller and auxiliary nonlinearities $\mathcal{N}_{1}$ and $\mathcal{N}_{2}$.

\section{III.C.1. Auxiliary Nonlinearity $\mathcal{N}_{1}$}

Define the saturation function sat ${ }_{a}$ by

$$
\mathcal{N}_{1}\left(u_{\mathrm{c}}\right)=\operatorname{sat}_{a}\left(u_{\mathrm{c}}\right)= \begin{cases}-a, & \text { if } u_{\mathrm{c}}<-a, \\ u_{\mathrm{c}}, & \text { if }-a \leq u_{\mathrm{c}} \leq a, \\ a, & \text { if } u_{\mathrm{c}}>a,\end{cases}
$$

where $a>0$ is the saturation level. For minimum-phase plants, the auxiliary nonlinearity $\mathcal{N}_{1}$ is not needed, and thus the saturation level $a$ is chosen to be a large number. For NMP plants, the saturation level $a$ is used to tune the transient behavior. In addition, the saturation level is chosen to provide the magnitude of the control input needed to follow the command $r$. This level depends on the range of the input nonlinearity $\mathcal{N}$ as well as the gain of the transfer function $G$ at frequencies in the spectra of $r$.

\section{III.C.2. Auxiliary Nonlinearity $\mathcal{N}_{2}$}

To construct $\mathcal{N}_{2}$, we assume that the intervals of monotonicity of the input nonlinearity $\mathcal{N}$ are known; no further modeling information about $\mathcal{N}$ is needed. Since the range of $\mathcal{N}_{1}$ is $[-a, a]$, we need consider only 
$u_{\mathrm{r}} \in[-a, a]$. Therefore, let $I_{1}, I_{2}, \ldots$ be intervals that partition the interval $[-a, a]$. If $\mathcal{N}$ is nondecreasing on $I_{i}$, then $\mathcal{N}_{2}\left(u_{\mathrm{r}}\right) \triangleq u_{\mathrm{r}}$ for all $u_{\mathrm{r}} \in I_{i}$. Alternatively, if $\mathcal{N}$ is nonincreasing on $I_{i}=\left(p_{i}, q_{i}\right)$, then $\mathcal{N}_{2}\left(u_{\mathrm{r}}\right) \triangleq$ $p_{i}+q_{i}-u_{\mathrm{r}} \in I_{i}$ for all $u_{\mathrm{r}} \in I_{i}$. Finally, if $\mathcal{N}$ is constant on $I_{i}$, then either choice can be used. Thus, $\mathcal{N}_{2} \circ \mathcal{N}$ is a piecewise-linear function that effectively replaces $\mathcal{N}$ by its mirror image on each interval within which $\mathcal{N}$ is nonincreasing. Let $\mathcal{R}_{a}(f)$ denote the range of the function $f$ with arguments in $[-a, a]$. Assume that $\mathcal{N}_{2}$ is constructed by the above rule. Then the following statements hold:

i) $\mathcal{N} \circ \mathcal{N}_{2}$ is piecewise nondecreasing.

ii) $\mathcal{R}_{a}\left(\mathcal{N} \circ \mathcal{N}_{2}\right)=\mathcal{R}_{a}(\mathcal{N})$.

Knowledge of only the intervals of monotonicity of $\mathcal{N}$ is needed to modify the controller output $u_{\mathrm{r}}$ so that $\mathcal{N} \circ \mathcal{N}_{2}$ is piecewise nondecreasing. For details, see. ${ }^{19}$

\section{III.C.3. NARMAX/O}

To choose nonlinear function $f_{j}$ for NARMAX/O controller

$$
u(k)=\sum_{j=1}^{p} \sum_{i=1}^{n_{\mathrm{c}}} M_{j i}(k) f_{j}(u(k-i))+\sum_{j=1}^{q} \sum_{i=1}^{n_{\mathrm{c}}} N_{j i}(k) z(k-i),
$$

we assume that the interval of controller outputs $\left[u_{\min }, u_{\max }\right]$ needed to follow the command signal $r$ is known and $\mathcal{N} \circ \mathcal{N}_{2}$ is piecewise nondecreasing. No further modeling information about $\mathcal{N}$ is required. The function $f(u)$ is chosen to be one-to-one for $u \in\left[u_{\min }, u_{\max }\right]$ and satisfies $f(0)=0$ if $0 \in\left[u_{\min }, u_{\max }\right]$.

\section{Numerical Examples}

Each example is constructed such that the first nonzero Markov parameter $H_{d}=1$, where $d$ is the relative degree of $G$. RCAC generates a control signal $u(k)$ that attempts to minimize the performance $z(k)$ in the presence of the reference signal $r$ and the input nonlinearity $\mathcal{N}$. We assume that measurements of $z(k)$ are available for feedback; however, measurements of $v=\mathcal{N}(u)$ are not available. In all cases, we initialize the adaptive controller to zero, that is, $\theta(0)=0$. We do not use a forgetting factor in this paper, that is, $\lambda=1$ for all examples.

To illustrate the distortion reduction on the closed-loop command-following performance with NARMAX RCAC controllers, we first simulate the Hammerstein plant with a linear RCAC controller. Following the same procedure, we simulate the Hammerstein plant using a NARMAX RCAC controller. Then, we compare the command-following performance $z$ for both cases. In all simulations, we run the open-loop system for the first 100 time steps. Then, at $k=100$, we turn the adaptation on, and let RCAC adapt the NARMAX controller in the presence of the unknown input nonlinearity $\mathcal{N}$.

Example IV.1. We consider the asymptotically stable, minimum-phase plant

$$
G(z)=\frac{(z-0.5)(z-0.9)}{(z-0.7)(z-0.5-\jmath 0.5)(z-0.5+\jmath 0.5)},
$$

with the input nonlinearity

$$
\mathcal{N}(u)=u^{3} .
$$

We consider the sinusoidal command $r(k)=\sin \left(\theta_{1} k\right)$, where $\theta_{1}=\pi / 5 \mathrm{rad} / \mathrm{sample}$. As shown in Figure $3(\mathrm{e})$, the input nonlinearity $\mathcal{N}$ is one-to-one and onto. We choose $\mathcal{N}_{1}\left(u_{\mathrm{c}}\right)=\operatorname{sat}_{a}\left(u_{\mathrm{c}}\right)$, where $a=10$ in (20). Since $\mathcal{N}$ is monotonically increasing for all $u \in \mathbb{R}$, we choose $\mathcal{N}_{2}\left(u_{\mathrm{r}}\right)=u_{\mathrm{r}}$. Note that knowledge of only 
the monotonicity of $\mathcal{N}$ is used to choose $\mathcal{N}_{1}$ and $\mathcal{N}_{2}$. We consider the NARMAX/O controller structure. In particular, we choose $f_{1}(u)=u, f_{2}(u)=\sin (2 u), f_{3}(u)=\sin (u), f_{4}(u)=\sin (0.5 u), f_{5}(u)=\sin (0.25 u)$, and $f_{6}(u)=\sin (0.125 u)$ for the NARMAX/O model. Furthermore, we let $n_{\mathrm{c}}=10, P_{0}=0.01 I_{8 n_{\mathrm{c}}}, \eta_{0}=0.001$, and $\tilde{\mathcal{H}}=H_{1}$. Figure 3 (a) and (b) show the closed-loop response of command-following performance $z$ and $\log |z|$ with a linear controller structure, and (c) and (d) show the closed-loop response with a NARMAX/O controller structure. The command-following performance in (c) and (d) are improved by approximately a two orders of magnitude compared with the performance in (a) and (b) at $k=2000$ time step. The NARMAX controller provides faster convergence compared with the linear controller structure. Figure 3(f) shows the auxiliary nonlinearity $\mathcal{N}_{2}\left(u_{\mathrm{r}}(k)\right)$. Finally, Figures $3(\mathrm{~g})$ and $(\mathrm{h})$ show the time history of the controller output $u$ and the controller gain vector $\theta$.

Example IV.2. We consider the linear plant (22) with the input nonlinearity $\mathcal{N}(u)=u^{3}+5$ and note that $\mathcal{N}(0) \neq 0$. We choose $f_{1}(u)=u$ and $f_{2}(u)=\sin (0.25 u)$ for the NARMAX/O model. We consider the same sinusoidal command $r(k)=\sin \left(\theta_{1} k\right)$, where $\theta_{1}=\pi / 5 \mathrm{rad} /$ sample and apply the same control parameters as in Example IV.1. We let the simulation run for 12, 000 time steps, and Figure 4(a) shows the closed-loop response of $\log |z|$ with the linear controller structure (dotted line) and NARMAX/O structure (solid line). NARMAX controller improves the steady-state performance.

Next, we further investigate the effect of the condition $\mathcal{N}(0) \neq 0$. Consider the linear plant (22) with the input nonlinearity $\mathcal{N}(u)=u^{3}+\varepsilon$, where $\varepsilon \in \mathbb{R}$. Furthermore, we consider same sinusoidal command $r(k)=\sin \left(\theta_{1} k\right)$, where $\theta_{1}=\pi / 5 \mathrm{rad} / \mathrm{sample}$, and apply the same NARMAX/O controller structure and controller parameters as in the last example. Figure 4(b) shows that the steady state performance of linear controller versus the NARMAX controller for various $\varepsilon$. Note that the NARMAX controller compensates for $\mathcal{N}(0) \neq 0$.

Example IV.3. We consider the asymptotically stable, nonminimum-phase plant

$$
G(z)=\frac{z-1.5}{(z-0.8)(z-0.6)},
$$

with the input nonlinearity

$$
\mathcal{N}(u)=-0.8 \tanh (u) .
$$

We consider the two-tone sinusoidal command $r(k)=0.5 \sin \left(\theta_{1} k\right)+0.5 \sin \left(\theta_{2} k\right)$, where $\theta_{1}=\pi / 5 \mathrm{rad} / \mathrm{sample}$ and $\theta_{2}=\pi / 2 \mathrm{rad} / \mathrm{sample}$. As shown in Figure $5(\mathrm{e})$, the input nonlinearity $\mathcal{N}_{\mathrm{H}}$ is one-to-one but not onto. We choose $\mathcal{N}_{1}\left(u_{\mathrm{c}}\right)=\operatorname{sat}_{a}\left(u_{\mathrm{c}}\right)$, where $a=2$ in (20). Since $\mathcal{N}$ is monotonically decreasing for all $u \in \mathbb{R}$, we choose $\mathcal{N}_{2}\left(u_{\mathrm{r}}\right)=-u_{\mathrm{r}}$. Note that knowledge of only the monotonicity of $\mathcal{N}$ is used to choose $\mathcal{N}_{1}$ and $\mathcal{N}_{2}$. We consider the NARMAX/O controller structure. In particular, we choose $f_{1}(u)=u, f_{2}(u)=u^{3}$, and $f_{3}(u)=u^{5}$ for the NARMAX/O model. Furthermore, we let $n_{\mathrm{c}}=10, P_{0}=0.01 I_{8 n_{\mathrm{c}}}, \eta_{0}=0.8$, and $\tilde{\mathcal{H}}=H_{1}$. Figures 5(a) and (b) show the closed-loop response of command-following performance $z$ and $\log |z|$ with a linear controller structure, and (c) and (d) show the closed-loop response with a NARMAX/O controller structure. The command-following performance in (c) and (d) are improved by approximately a one order of magnitude compared with the performance in (a) and (b). Figure $5(\mathrm{f})$ shows the auxiliary nonlinearity $\mathcal{N}_{2}\left(u_{\mathrm{r}}(k)\right)$. Finally, Figures $5(\mathrm{~g})$ and $(\mathrm{h})$ show the time history of the controller output $u$ and the controller gain vector $\theta$.

Example IV.4. We consider the asymptotically stable, minimum-phase plant

$$
G(z)=\frac{(z-0.5)(z-0.9)}{(z-0.7)(z-0.5-\jmath 0.5)(z-0.5+\jmath 0.5)},
$$

with the input nonlinearity

$$
\mathcal{N}_{\mathrm{H}}(u)=\cos (u) .
$$

We consider the sinusoidal command $r(k)=\sin \left(\theta_{1} k\right)$, where $\theta_{1}=\pi / 5 \mathrm{rad} /$ sample. As shown in Figure $6(\mathrm{e})$, the input nonlinearity $\mathcal{N}$ is neither one-to-one nor onto. We choose $\mathcal{N}_{1}\left(u_{\mathrm{c}}\right)=\operatorname{sat}_{a}\left(u_{\mathrm{c}}\right)$, where $a=4$ 


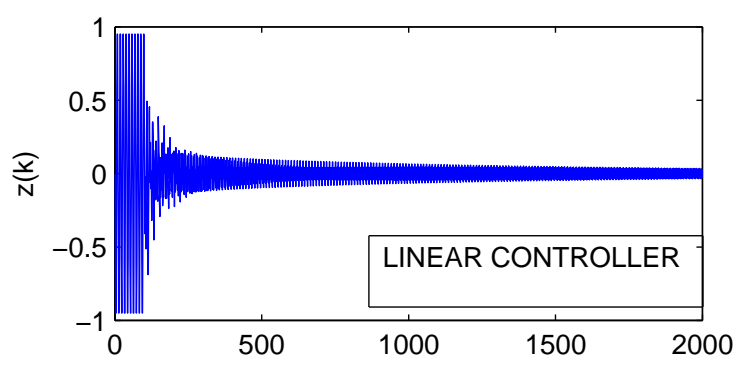

(a)

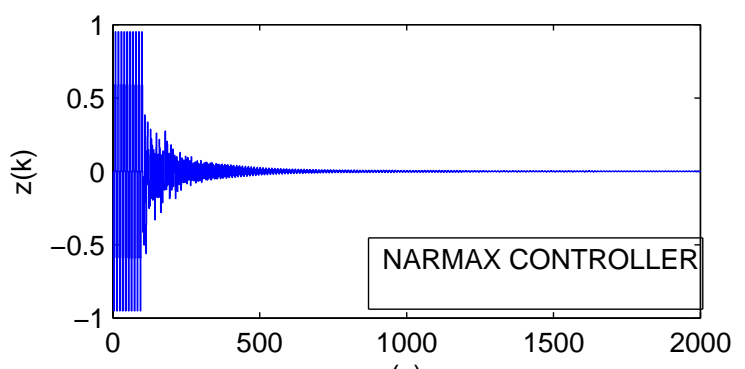

(c)

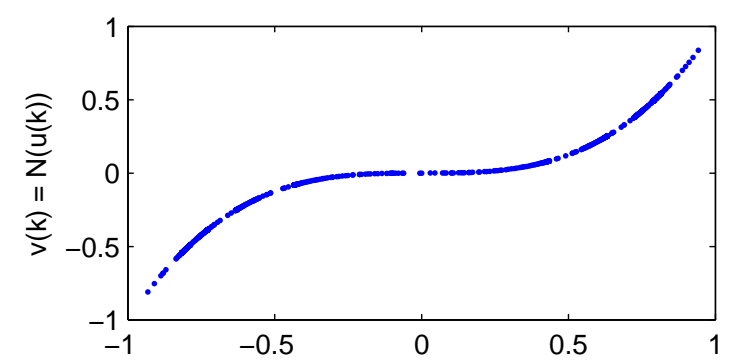

(e) $u(k)$

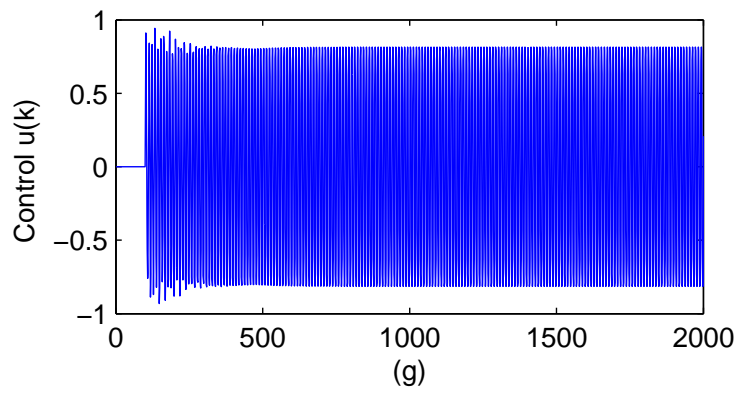

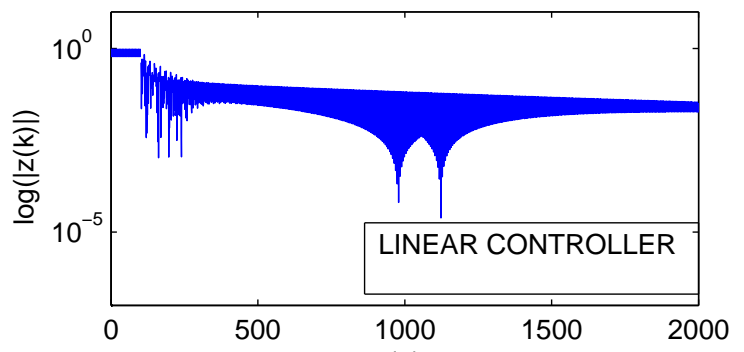

(b)

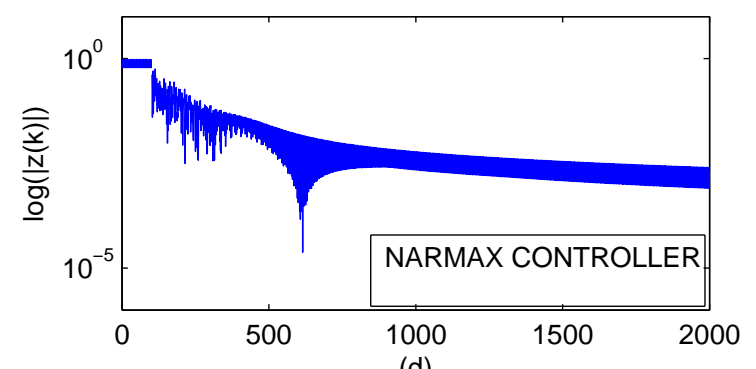

(d)

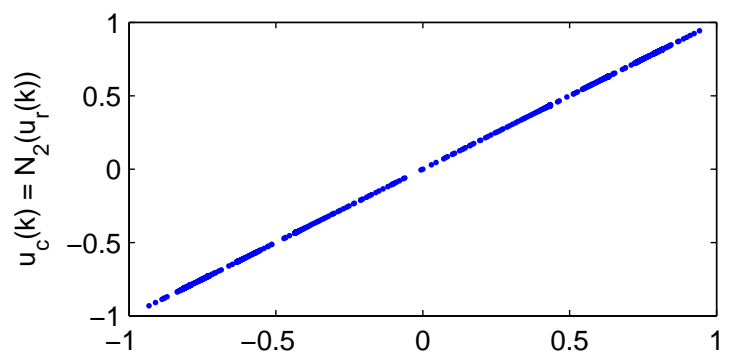

(f) $u_{r}(k)$

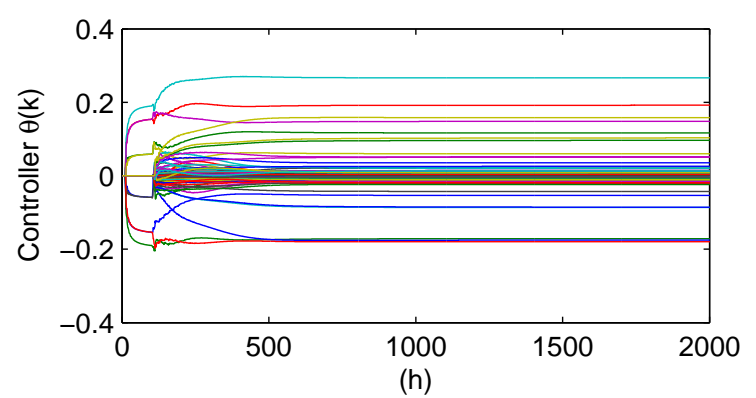

Figure 3. Example IV.1. Closed-loop response of the plant $G$ given by (22). The system runs open loop for 100 time steps, and the adaptive controller is turned on at $k=100$ with auxiliary nonlinearity $\mathcal{N}_{1}$ and $\mathcal{N}_{2}$ based on the knowledge the monotonicity of $\mathcal{N}$. The plant output $y$ follows the command $r(k)$. We choose $f_{1}(u)=u, f_{2}(u)=\sin (2 u)$, $f_{3}(u)=\sin (u), f_{4}(u)=\sin (0.5 u), f_{5}(u)=\sin (0.25 u)$, and $f_{6}(u)=\sin (0.125 u)$ for the NARMAX/O model. The NARMAX controller provides faster convergence compared with the linear RCAC, and (a) and (b) show the closed-loop response of command-following performance $z$ and $\log |z|$ with a linear controller structure. The command-following performance in (c) and (d) are improved by approximately a two orders of magnitude compared with the performance in (a) and (b) at $k=2000$ time step.

in (20). Since $\mathcal{N}$ is monotonically increasing for all $u \in(2 n \pi-\pi, 2 n \pi), n \in \mathbb{Z}$, we choose $\mathcal{N}_{2}\left(u_{\mathrm{r}}\right)=u_{\mathrm{r}}$, and 


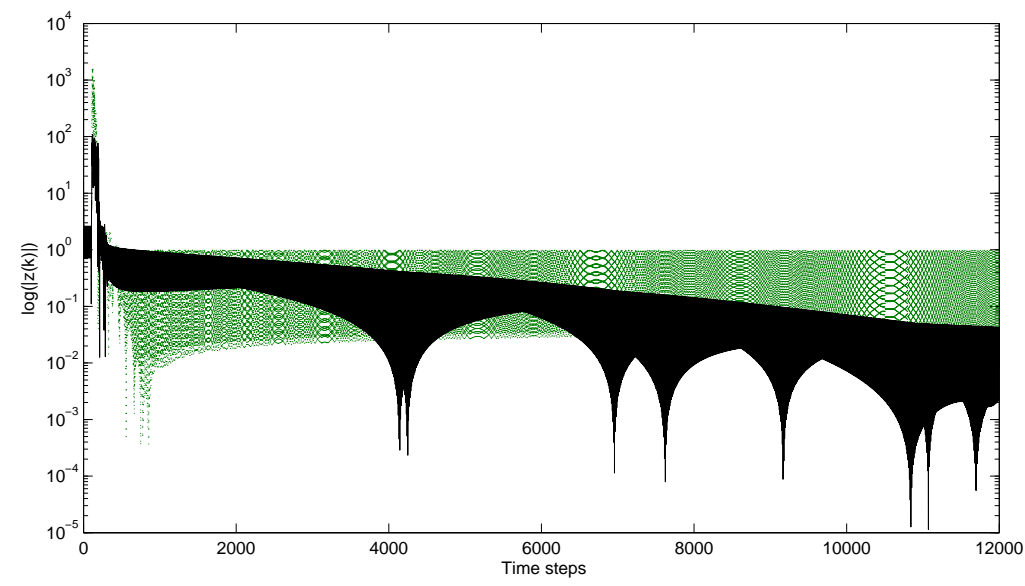

(a)

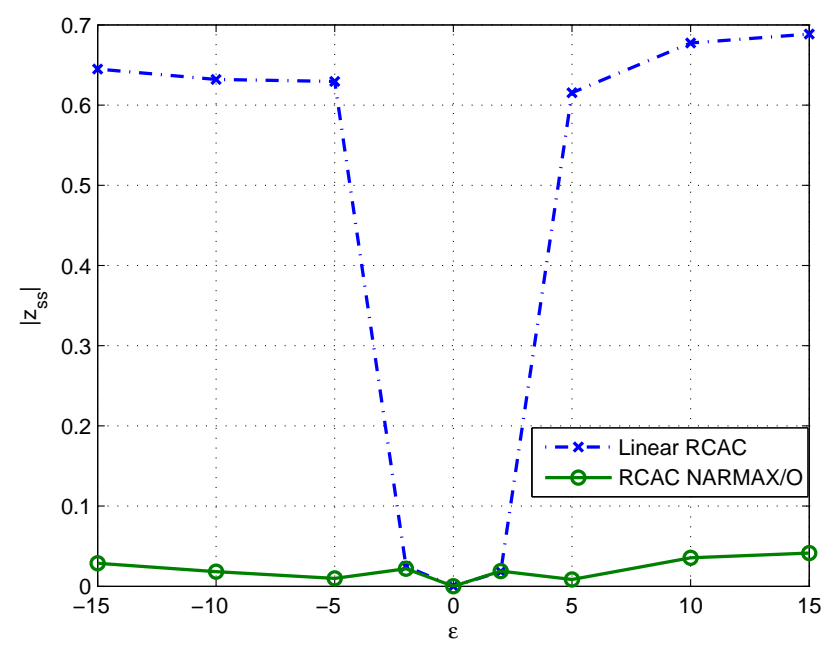

(b)

Figure 4. Example IV.2. (a) shows the closed-loop response to the command signal $r(k)=\sin \left(\theta_{1} k\right)$, where $\theta_{1}=\pi / 5$ $\mathrm{rad} /$ sample with the nonlinearities $\mathcal{N}(u)=u^{3}+5$ and the linear plant (22) with the linear controller structure (solid line) and NARMAX/O controller (dotted line). NARMAX controller improves the steady state performance with input nonlinearity satisfying $\mathcal{N}(0) \neq 0$. (b) shows that the steady state performance of linear controller versus the NARMAX controller for various $\varepsilon$. Note that $\left|z_{\mathrm{ss}}\right|=0.707$ is the performance error for the open-loop system and the command-following performance distortion is compensated by the NARMAX controller with the input nonlinearity satisfying $\mathcal{N}(0)=\varepsilon$.

since $\mathcal{N}$ is monotonically decreasing for all $u \in(2 n \pi, 2 n \pi+\pi), n \in \mathbb{Z}$, we choose $\mathcal{N}_{1}\left(u_{\mathrm{c}}\right)=-u_{\mathrm{r}}+(4 n+$ 1) $\pi$. Note that knowledge of only the monotonicity of $\mathcal{N}$ is used to choose $\mathcal{N}_{1}$ and $\mathcal{N}_{2}$. We consider the NARMAX/IO controller structure. In particular, we choose $g_{1}(z)=z, g_{2}(z)=\sin (2 z), g_{3}(z)=\sin (z)$, $g_{4}(z)=\sin (0.5 z), f_{1}(u)=u, f_{2}(u)=\sin (2 u), f_{3}(u)=\sin (u)$, and $f_{4}(u)=\sin (0.5 u)$ for the NARMAX/IO controller. Furthermore, we let $n_{\mathrm{c}}=10, P_{0}=0.1 I_{14 n_{\mathrm{c}}}, \eta_{0}=0.02$, and $\tilde{\mathcal{H}}=H_{1}$. Figures 6 (a) and (b) show the closed-loop response with a linear RCAC, and (c) and (d) show the closed-loop response with a NARMAX RCAC controller. The command-following distortion in (c) and (d)are approximately $20 \%$ smaller compared with the performance distortion in (a) and (b). Figure 6(f) shows the auxiliary nonlinearity $\mathcal{N}_{2}\left(u_{\mathrm{r}}(k)\right)$. Figures $6(\mathrm{~g})$ and $(\mathrm{h})$ show the time history of the controller output $u$ and the controller gain vector $\theta$. Finally, Figure 6(i) shows the time history of the control $u_{\mathrm{r}}$ and the plant input $v$ and $v$ is piecewise increasing versus $u_{\mathrm{r}}$. 


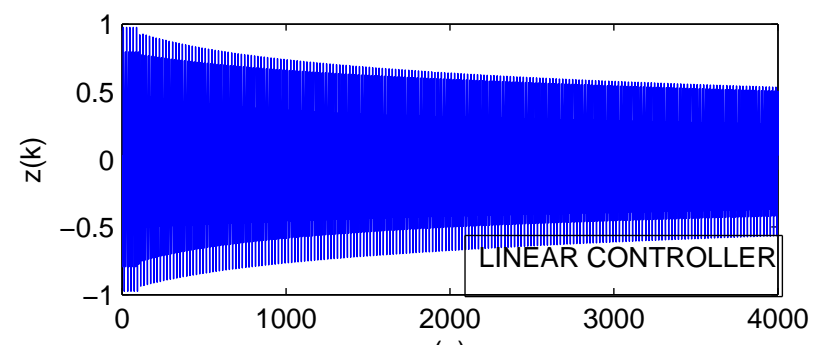

(a)

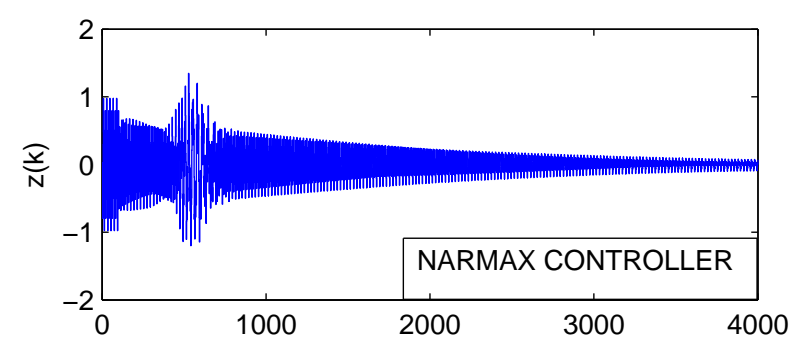

(c)

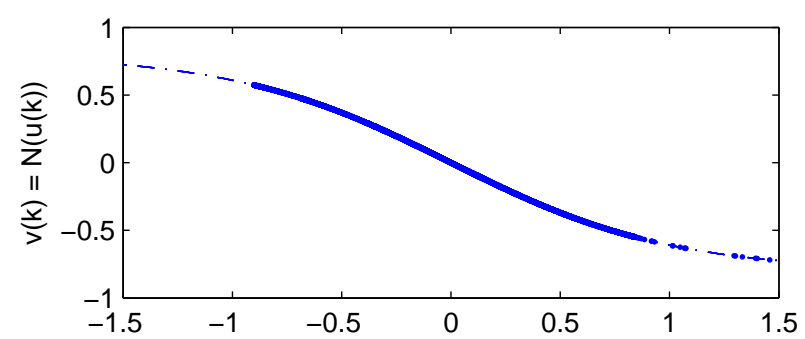

(e) $u(k)$

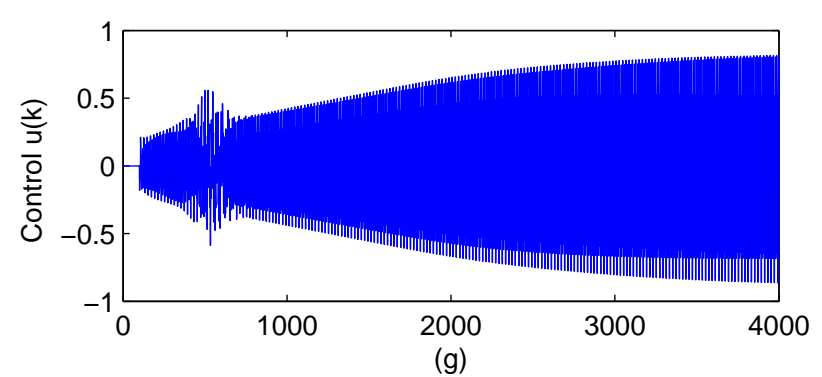

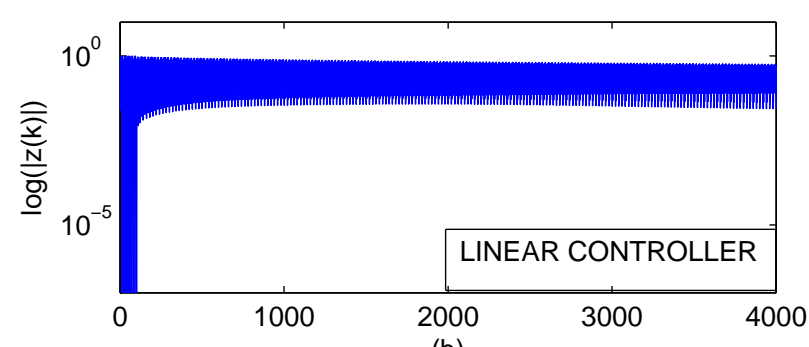

(b)

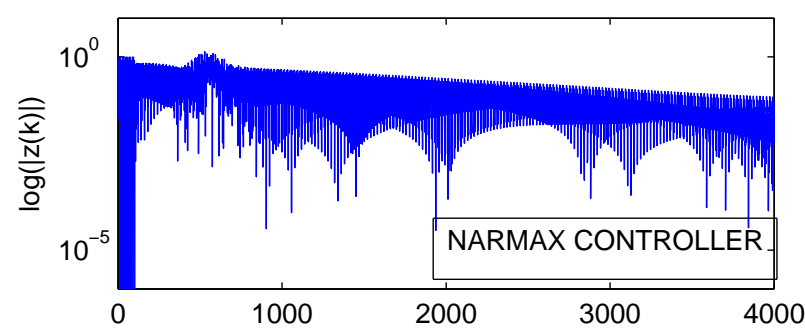

(d)

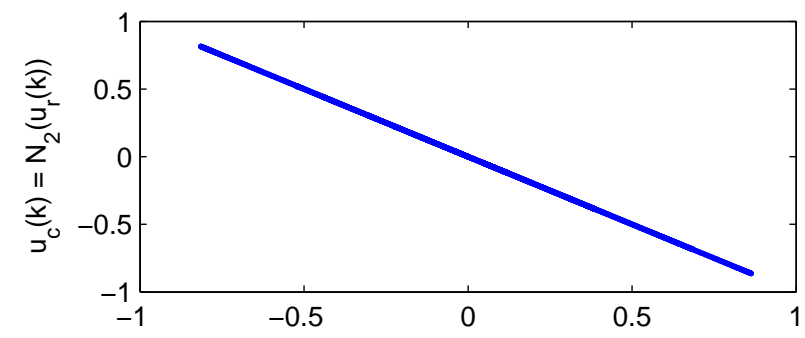

(f) $u_{r}(k)$

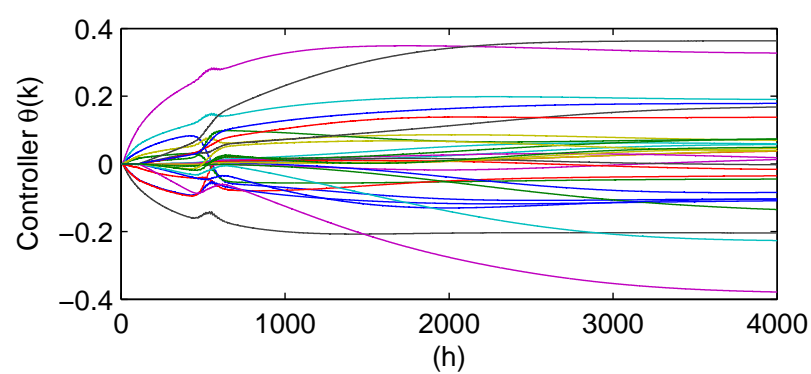

Figure 5. Example IV.3. Closed-loop response of the plant $G$ given by (24). The system runs open loop for 500 time steps, and the adaptive controller is turned on at $k=500$ with input nonlinearity $\mathcal{N}_{1}$ and $\mathcal{N}_{2}$ based on the knowledge the monotonicity of $\mathcal{N}$. The plant output $y_{0}$ follows the command $r(k)$. We choose $f_{1}(u)=u, f_{2}(u)=u^{3}$, and $f_{3}(u)=u^{5}$ for the NARMAX/O model. Figure 5 (a) and (b) show the closed-loop response of command-following performance $z$ and $\log |z|$ with a linear controller structure. The command-following performance in (c) and (d) are improved by approximately a one order of magnitude compared with the performance in (a) and (b).

\section{Conclusions}

Retrospective cost adaptive control (RCAC) with a NARMAX control structure was applied to command following for Hammerstein systems. RCAC was used with limited modeling information. In particular, 


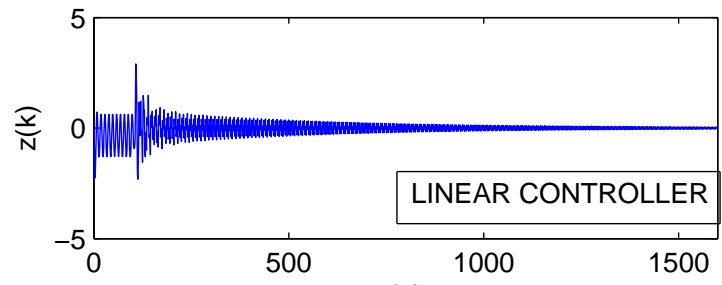

(a)

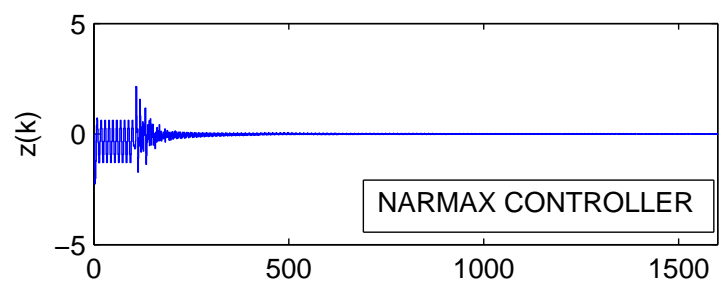

(c)

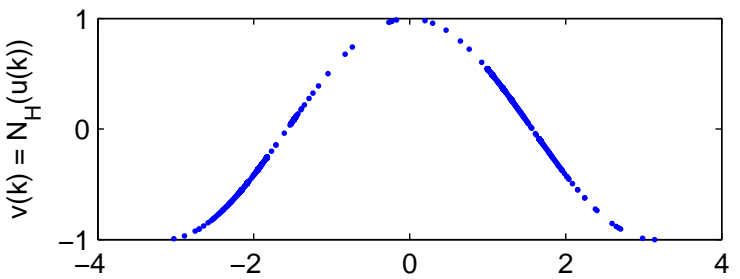

(e) u(k)

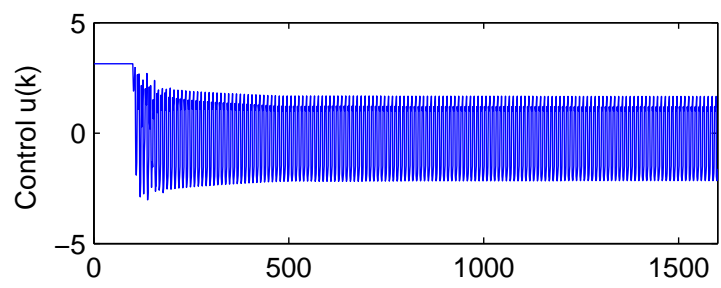

(g)

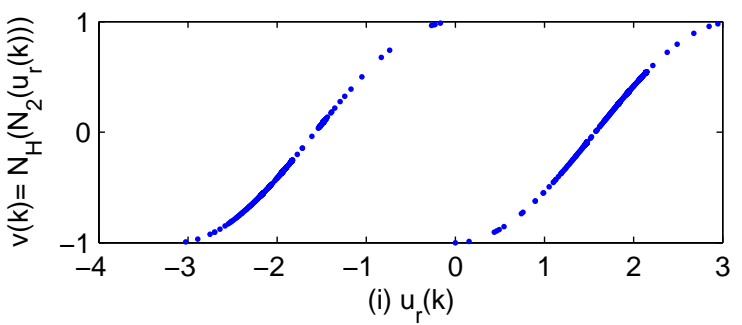

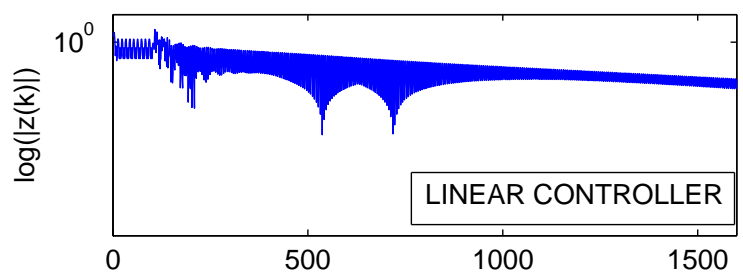

(b)

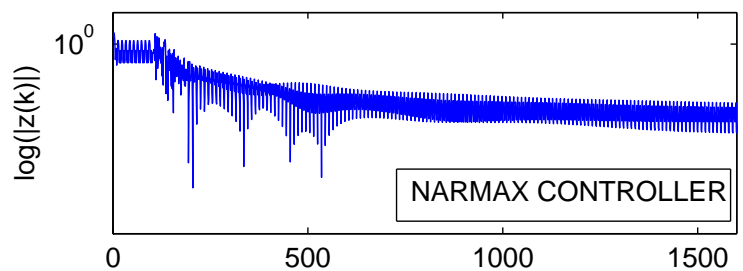

(d)

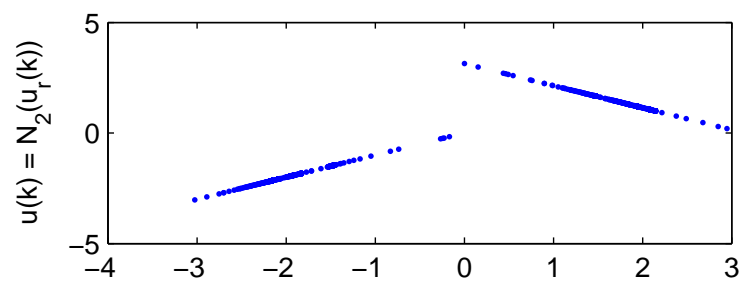

(f) $u_{r}(k)$

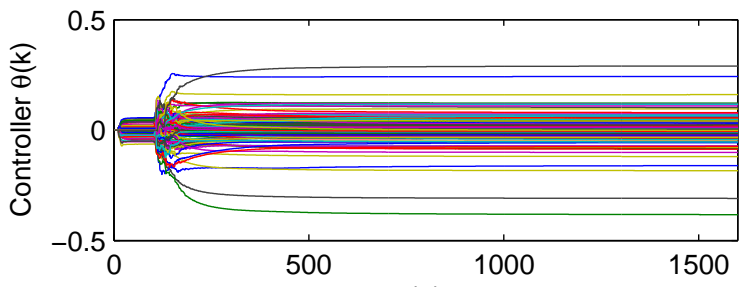

(h)

Figure 6. Example IV.4. Closed-loop response of the plant $G$ given by (26). The system runs open loop for 500 time steps, and the adaptive controller is turned on at $k=500$ with input nonlinearity $\mathcal{N}_{1}$ and $\mathcal{N}_{2}$ based on the knowledge the monotonicity of $\mathcal{N}$. The plant output $y_{0}$ follows the command $r(k)$. We choose $g_{1}(z)=z, g_{2}(z)=\sin (2 z), g_{3}(z)=\sin (z)$, $g_{4}(z)=\sin (0.5 z), f_{1}(u)=u, f_{2}(u)=\sin (2 u), f_{3}(u)=\sin (u)$, and $f_{4}(u)=\sin (0.5 u)$ for the NARMAX/IO controller. Figures 6(a) and (b) show the closed-loop response with a linear controller structure. The command-following performance distortion in (c) and (d) are approximately $20 \%$ smaller compared with the performance distortion in (a) and (b) using the NARMAX/IO controller structure. 
RCAC uses knowledge of the first nonzero Markov parameter of the linear system. To handle the effect of the input nonlinearity, RCAC was augmented by auxiliary nonlinearities chosen based on the monotonicity properties of the input nonlinearity. We numerically demonstrated that RCAC with a NARMAX controller structure can improve the command-following performance for the Hammerstein systems over the linear controller structure for providing fast convergence and compensating performance distortion for $\mathcal{N}(0) \neq 0$. Future research will focus on choosing NARMAX structures for RCAC based on limited knowledge of the uncertain Hammerstein nonlinearities.

\section{References}

${ }^{1}$ D. S. Bernstein and W. M. Haddad, "Nonlinear controllers for positive real systems with arbitrary input nonlinearities," IEEE Transactions on Automatic Control, vol. 39, pp. 15131517, 1994

${ }^{2}$ W. Haddad and V. Chellaboina, "Nonlinear control of hammerstein systems with passive nonlinear dynamics," IEEE Transactions on Automatic Control, vol. 46, no. 10, pp. 1630 - 1634, 2001.

${ }^{3}$ G. Tao and M. Tian, "Discrete-time adaptive control of systems with multisegment piecewise-linear nonlinearities," IEEE Trans. Autom. Contr., vol. 43, no. 5, pp. 719-723, 1998.

${ }^{4}$ E. D. Sumer, A. M. D'Amato, A. M. Morozov, J. B. Hoagg, and D. S. Bernstein, "Robustness of Retrospective Cost Adaptive Control to Markov-Parameter Uncertainty," Proc. Conf. Dec. Contr., pp. 6085-6090, Orlando, FL, December 2011.

${ }^{5}$ G. Tao, Adaptive Control of Systems with Actuator and Sensor Nonlinearities, Wiley, 1996.

${ }^{6}$ M. Aljanaideh, D. Sumer, J. Yan, A. M. D'Amato, B. Drincic, K. Aljanaideh, and D. S. Bernstein, "Adaptive Control of Uncertain Hammerstein Systems with Uncertain Hysteretic Input Nonlinearities", Proc. ASME Dynamic Systems and Controls Conference, Fort Lauderdale, FL, October 2012.

${ }^{7}$ J. Yan, A. M. D'Amato, and D. S. Bernstein, "Setpoint Control of the Uncertain Electromagnetically Controlled Oscillator", Proc. ASME Dynamic Systems and Controls Conference, Fort Lauderdale, FL, October 2012.

${ }^{8}$ M. Aljanaideh, J. Yan, A. M. D'Amato, and D. S. Bernstein, "Retrospective-Cost Adaptive Control of Uncertain Wiener-Hammerstein Systems with Memoryless and Hysteretic Nonlinearities," AIAA Guid. Nav. Contr. Conf., Minneapolis, MN, August 2012.

${ }^{9}$ R. Venugopal and D. S. Bernstein, "Adaptive Disturbance Rejection Using ARMARKOV System Representations," IEEE Trans. Contr. Sys. Tech., Vol. 8, pp. 257-269, 2000.

${ }^{10}$ J. B. Hoagg, M. A. Santillo, and D. S. Bernstein, "Discrete-Time Adaptive Command Following and Disturbance Rejection with Unknown Exogenous Dynamics," IEEE Trans. Autom. Contr., Vol. 53, pp. 912-928, 2008.

${ }^{11}$ M. A. Santillo and D. S. Bernstein, "Adaptive Control Based on Retrospective Cost Optimization," AIAA J. Guid. Contr. Dyn., Vol. 33, pp. 289-304, 2010.

12 J. B. Hoagg and D. S. Bernstein, "Retrospective Cost Model Reference Adaptive Control for Nonminimum-Phase Discrete-Time Systems, Part 1: The Adaptive Controller; Part 2: Stability Analysis," Proc. Amer. Contr. Conf., pp. 2927-2938, San Francisco, CA, June 2011.

${ }^{13}$ A. M. D'Amato, E. D. Sumer, and D. S. Bernstein, "Retrospective Cost Adaptive Control for Systems with Unknown Nonminimum-Phase Zeros," AIAA Guid. Nav. Contr. Conf., Portland, OR, August 2011, AIAA-2011-6203.

${ }^{14}$ A. M. D'Amato, E. D. Sumer, and D. S. Bernstein, "Frequency-Domain Stability Analysis of Retrospective-Cost Adaptive Control for Systems with Unknown Nonminimum-Phase Zeros," Proc. Conf. Dec. Contr., pp. 1098-1103, Orlando, FL, December 2011.

${ }^{15}$ S. Chen, S. A. Billings, C. F. N. Cowan and P. M. Grant, "Practical identification of NARMAX models using radial basis function," Int. J. Control, Vol. 52, No.6, pp. 1327-1350, 1990.

${ }^{16}$ S. Chen and S. A. Billings, "Representation of non-linear systems: the NARMAX model," Int. J. Control, Vol. 49, No.3, pp.1013-1032, 1989.

${ }^{17}$ S. L. Kukreja, H. L. Galiana and R. E. Kearney, "A bootstrap method for structure detection of NARMAX models," Int. J. Control, Vol. 77, No.2, pp. 132-143, 2004.

${ }^{18}$ T. A. Johansen and B. A. Foss, "A NARMAX model representation for adaptive control based on local models," Modeling, Identification And Control, Vol. 13, No.1, pp. 25-39, 1992.

${ }^{19}$ J. Yan, A. M. D'Amato, E. D. Sumer, J. B. Hoagg, and D. S. Bernstein, "Adaptive Control of Uncertain Hammerstein Systems Using Auxiliary Nonlinearities," Proc. Conf. Dec. Contr., Maui, HI, December 2012. 